\title{
Trends in Suspected Opioid Overdoses from Emergency Departments in 11 States and DC
}

\author{
Stephen Liu, Matthew Gladden, ALANA M. VIVOLO-KANTOR, Puja Seth
}

Centers for Disease Control and Prevention (CDC), Atlanta, Georgia, United States

Objective

This presentation will provide insight into how the extensive spread of illicitly-manufactured fentanyl impacted opioid overdose rates throughout the Midwest and neighboring states.

\section{Introduction}

Recent reporting using data from CDC's National Syndromic Surveillance Program indicates that rates of emergency department (ED) visits involving suspected opioid overdoses increased by 70\% in the Midwest from the third quarter (Q3) 2016 (JulySeptember) to the Q3 2017. Large increases in the use and distribution of illicitly-manufactured fentanyl (IMF) and fentanyl analogs, are a key factor driving increased opioid overdose rates in the Midwest and east of the Mississippi River. Fentanyl is a synthetic opioid 50-100 times more potent than morphine. A better understanding of the distribution of changes in opioid overdose rate from Q3 2016 to Q3 2017 within states needed to inform response and prevention efforts.

\section{Methods}

The CDC's Enhanced State Opioid Overdose Surveillance Program currently funds 32 states and Washington DC to increase timeliness of opioid overdose reporting and detect rapid changes in trends. Data from nine states (IL, MD, MO, NC, OH, PA, VA, WI, WV) were analyzed. Midwest states sharing subregional data with CDC were selected to better understand geographic and temporal patterns driving previously reported increases in ED visits involving suspected opioid overdoses from Q3 2016 through Q3 2017. Bordering states (MD, NC, PA, VA, WV) sharing subregional data with CDC were also included to determine trends in states contiguous to the Midwest. State subregions were defined using publicly available state government sources in consultation with state public health departments and were mainly divided by public health districts. . Fifty of 56 possible state subregions across 9 states met two inclusion criteria: 1) reported 25 opioid overdose ED visits per quarter and 2) did not report a change of $50 \%$ between any two quarters. Opioid overdoses were defined according to jurisdictional and national definitions that included se arches of chief complaint text (e.g., searching for words "opioid" and "overdose") and ICD-10-CM diagnostic/billing codes. State subregional rates were defined as number of opioid overdoses divided by the total number of ED visits in the state subregion, multiplied by 10,000. Quarterly and yearly percent change in opioid overdose ED visits from Q3 2016 to Q3 2017 were described with a focus on high burden subregions reporting large yearly increases of 25\% from Q3 2016 to Q3 2017. We categorized a subregion as having an opioid overdose outbreak when at least one quarterly rate increase in opioid overdoses of $50 \%$ occurred.

\section{Results}

Over 7 million ED visits were reported each quarter. Average subregional opioid overdose rates increased at a consistent quarterly rate of between 16\% - 19\% during Q3 2016 to Q2 2017. From Q2 2017 to Q3 2017, opioid overdose rates only increased 1\%. Overall, subregions reported a mean yearly increase in opioid overdose rates of 51\% from Q3 2016 to Q3 2017. This yearly increase in opioid overdose from Q3 2016 to Q3 2017 was unequally distributed across subregions with 9 (18\%) of subregions reporting an increase in opioid overdose rates of 100\%, $13(26 \%)$ reporting an increase of 50\% - <100\%, $7(14 \%)$ reporting an increase of $25 \%$ - $<50 \%, 13(26 \%)$ reporting an increases of $0 \%$ to <25\%, and $8(16 \%)$ reporting a decrease. Analyses of the 29 high burden subregions found that $16(55 \%)$ reported at least one opioid overdose outbreak compared to 3 of 21 other subregions (14\%) (Table 1). The 16 high burden subregions that reported any outbreak had a mean yearly increase in opioid overdose rates from Q3 2016 to Q3 2017 of 107\%, range 33\% to 266\%. Eight of these 16 high burden subregions either reported two opioid overdose outbreaks or an opioid overdose outbreak and a quarterly increase of between $25 \%$ - <50\%. All states had at least one subregion report an opioid overdose outbreak between Q3 2016 to Q2 2017. Also, within OH, PA, WI, and WV, half or more of their subregions reported an outbreak between Q3 2016 and Q3 2017 (Table 1). Fifteen of the 22 (68\%) quarterly opioid overdose outbreaks occurred either during Q4 2016 or Q1 2017 (Table 1). Across all outbreaks, state subregions reported a mean quarterly increase of 83\% with a range from $50-156 \%$.

SDS Annual Conference Proceedings 2019. This is an Open Access article distributed under the terms of the Creative Commons AttributionNoncommercial 4.0 Unported License (http://creativecommons.org/licenses/by-nc/3.0/), permitting all non-commercial use, distribution, and reproduction in any medium, provided the original work is properly cited. 


\section{Conclusions}

Opioid overdose outbreaks in a subset of 16 high burden subregions across all 9 states were a key factor driving increases in the states' opioid overdose rates. Half of these 16 subregions experienced a single opioid overdose outbreak while the other half experienced two or more sharp increases. The majority of opioid overdose outbreaks occurred during October 2016 to March 2017 were concentrated in Ohio, a state reporting extremely large increase in IMF supply and overdose deaths involving fentanyl, and states contiguous with Ohio. Although most subregions reported outbreaks at the beginning of the study period, higher opioid overdose rates persisted in the vast majority of subregions reporting opioid overdose outbreaks. This outbreak pattern is consistent with previous findings showing large increases in the supply of IMF and fentanyl analogs, including carfentanil, in Midwestern states during 2016 and in early 2017. Although opioid overdose outbreaks were concentrated in subregions in OH, PA, and WV, all 9 states included in the analysis were impacted by at least one outbreak during the study period. Findings highlight the need for targeting of hotspots within states and implementation of public health interventions to reduce harm and surge resources during an outbreak. These short-term efforts, however, must be complemented by a sustained response to reduce increased drug overdose rates that persist after an initial outbreak. A key limitation of this study was that it only included data from a single year and as a result may underestimate the length and severity of outbreaks. As changes in the illicit opioid market continue, surveillance of local outbreaks must be supplemented by broader surveillance designed to detect both localized introduction of new novel psychoactive substances as well as large scale changes in the illicit opioid market.

\section{References}

1. Vivolo-Kantor AM, Seth P, Gladden RM, et al. 2018. Vital Signs: Trends in Emergency Department Visits for Suspected Opioid Overdoses_- United States, July 2016-September 2017. MMWR Morb Mortal Wkly Rep. 67, 279-85. PubMed https://doi.org/10.15585/mmwr.mm6709e1

2. O’Donnell J, Gladden RM, Mattson CL, Kariisa M. 2018. Notes from the Field: Overdose Deaths with Carfentanil and Other Fentanyl Analogs Detected - 10 States, July 2016-June 2017. MMWR Morb Mortal Wkly Rep. 67, 767-68. PubMed https://doi.org/10.15585/mmwr.mm6727a4

3. O’Donnell JK, Halpin J, Mattson CL, Goldberger BA, Gladden RM. 2017. Deaths Involving Fentanyl, Fentanyl Analogs, and U-47700 — 10 States, July-December 2016. MMWR Morb Mortal Wkly Rep. 66, 1197202. PubMed https://doi.org/10.15585/mmwr.mm6643e1

\begin{tabular}{|c|c|c|c|c|c|c|c|c|c|c|c|c|}
\hline & \multirow{2}{*}{\multicolumn{2}{|c|}{$\begin{array}{c}\text { Total } \\
\text { subregions }\end{array}$}} & \multicolumn{10}{|c|}{$\begin{array}{c}\text { " of subregions reporting opioid overdose outbreaks } \\
\text { ( } \geq 50 \% \text { quarterly change in opioid overdose rate) }\end{array}$} \\
\hline & & & \multicolumn{2}{|c|}{$\begin{array}{c}\text { All quarterly } \\
\text { changes from } \\
\text { Q3 2016-Q3 2017 }\end{array}$} & \multicolumn{2}{|c|}{ Q32016-Q4 2016} & \multicolumn{2}{|c|}{ Q4 2016-Q1 2017} & \multicolumn{2}{|c|}{ Q1 2017-Q2 2017} & \multicolumn{2}{|c|}{ Q2 2017-Q3 2017} \\
\hline & $\|$ & $\%$ & $\#$ & $\%$ & $\#$ & $\%$ & $\#$ & $\%$ & $\#$ & $\%$ & $\#$ & $\%$ \\
\hline \multicolumn{13}{|l|}{$\begin{array}{l}\text { \% change in } \\
\text { opioid overdose } \\
\text { rates from Q3 } \\
2016 \text { to Q3 } 2017\end{array}$} \\
\hline Rates declined & 8 & $16 \%$ & 1 & $13 \%$ & 0 & $0 \%$ & 0 & $0 \%$ & 1 & $13 \%$ & 0 & $0 \%$ \\
\hline $\begin{array}{l}\text { Rates increased } \\
\text { from } 0 \% \text { to } 25 \%\end{array}$ & 13 & $26 \%$ & 2 & $15 \%$ & 0 & $0 \%$ & 2 & $15 \%$ & 0 & $0 \%$ & 0 & $0 \%$ \\
\hline $\begin{array}{l}\text { High burden } \\
\text { (increased } 25 \% \text { or } \\
\text { more) }\end{array}$ & 29 & $58 \%$ & 16 & $55 \%$ & 9 & $31 \%$ & 4 & $14 \%$ & 4 & $14 \%$ & 2 & $7 \%$ \\
\hline & & & & & & & & & & & & \\
\hline \multicolumn{13}{|l|}{ States by region } \\
\hline \multicolumn{13}{|l|}{ Northeast } \\
\hline Pennsylvania & 6 & $12 \%$ & 3 & $50 \%$ & 1 & $17 \%$ & 1 & $17 \%$ & 1 & $17 \%$ & 1 & $17 \%$ \\
\hline \multicolumn{13}{|l|}{ Midwest } \\
\hline Illinois & 6 & $12 \%$ & 2 & $33 \%$ & 1 & $17 \%$ & 1 & $17 \%$ & 0 & $0 \%$ & 0 & $0 \%$ \\
\hline Missouri & 5 & $10 \%$ & 2 & $40 \%$ & 0 & $0 \%$ & 1 & $20 \%$ & 0 & $0 \%$ & 1 & $20 \%$ \\
\hline Ohio & 7 & $14 \%$ & 4 & $57 \%$ & 3 & $43 \%$ & 2 & $29 \%$ & 0 & $0 \%$ & 0 & $0 \%$ \\
\hline Wisconsin & 4 & $8 \%$ & 2 & $50 \%$ & 1 & $25 \%$ & 1 & $25 \%$ & 0 & $0 \%$ & 0 & $0 \%$ \\
\hline \multicolumn{13}{|l|}{ South } \\
\hline Maryland & 4 & $8 \%$ & 1 & $25 \%$ & 0 & $0 \%$ & 0 & $0 \%$ & 1 & $25 \%$ & 0 & $0 \%$ \\
\hline North Carolina & 9 & $18 \%$ & 1 & $5 \%$ & 0 & $0 \%$ & 0 & $0 \%$ & 1 & $11 \%$ & 0 & $0 \%$ \\
\hline Virginia & 5 & $10 \%$ & 1 & $20 \%$ & 0 & $0 \%$ & 0 & $0 \%$ & 1 & $20 \%$ & 0 & $0 \%$ \\
\hline West Virginia & 4 & $8 \%$ & 3 & $75 \%$ & 3 & $75 \%$ & 0 & $0 \%$ & 1 & $25 \%$ & 0 & $0 \%$ \\
\hline All subregions & 50 & $100 \%$ & 19 & $38 \%$ & 9 & $18 \%$ & 6 & $12 \%$ & 5 & $10 \%$ & 2 & $4 \%$ \\
\hline
\end{tabular}

Table 1. Number and percent of opioid overdose outbreaks by quarter, high burden subregions, and state: 9 states July 2016 to September 2017 